\title{
DOCK7 is a critical regulator of the RAGE-Cdc42 signaling axis that induces formation of dendritic pseudopodia in human cancer cells
}

\author{
KEN-ICHI YAMAMOTO ${ }^{1}$, HITOSHI MURATA ${ }^{1}$, ENDY WIDYA PUTRANTO ${ }^{1}$, KEN KATAOKA ${ }^{2}$, \\ AKIRA MOTOYAMA $^{3}$, TOSHIHIKO HIBINO ${ }^{3}$, YUSUKE INOUE $^{4}$, MASAKIYO SAKAGUCHI $^{1}$ and NAM-HO HUH ${ }^{1}$ \\ ${ }^{1}$ Department of Cell Biology, Okayama University Graduate School of Medicine, Dentistry and Pharmaceutical Sciences, \\ Kita-ku, Okayama 700-8558; ${ }^{2}$ Department of Life Science, Faculty of Science, Okayama University of Science, \\ Kita-ku, Okayama 700-0005; ${ }^{3}$ Shiseido Research Center, Kanazawa-ku, Yokohama 236-8643; \\ ${ }^{4}$ Department of Chemistry and Chemical Biology, Graduate School of Engineering, \\ Gunma University, Kiryu, Gunma 376-8515, Japan
}

Received August 10, 2012; Accepted October 25, 2012

DOI: $10.3892 /$ or.2012.2191

\begin{abstract}
Cellular migration is a fundamental process linked to cancer metastasis. Growing evidence indicates that the receptor for advanced glycation end products (RAGE) plays a pivotal role in this process. With regard to downstream signal transducers of RAGE, diaphanous-1 and activated small guanine nucleotide triphosphatases, $\mathrm{Rac1}$ and $\mathrm{Cdc} 42$, have been identified. To obtain precise insight into the direct downstream signaling mechanism of RAGE, we screened for proteins interacting with the cytoplasmic domain of RAGE employing an immunoprecipitation-liquid chromatography coupled with an electrospray tandem mass spectrometry system. In the present study, we found that the cytoplasmic domain of RAGE interacted with an atypical DOCK180-related guanine nucleotide exchange factor, dedicator of cytokinesis protein 7 (DOCK7). DOCK7 bound to the RAGE cytoplasmic domain and transduced a signal to $\mathrm{Cdc} 42$, resulting in the formation of abundant highly branched filopodia-like protrusions, dendritic pseudopodia. Blocking of the function of DOCK7 greatly abrogated the formation of dendritic pseudopodia and suppressed cellular migration. These results indicate that DOCK7 functions as an essential and downstream regulator
\end{abstract}

Correspondence to: Professor Nam-ho Huh, Department of Cell Biology, Okayama University Graduate School of Medicine, Dentistry and Pharmaceutical Sciences, 2-5-1 Shikatacho, Kita-ku, Okayama 700-8558, Japan

E-mail: namu@md.okayama-u.ac.jp

Abbreviations: RAGE, receptor for advanced glycation end products; DOCK7, dedicator of cytokinesis protein 7; Dia-1, diaphanous-1; GTPase, guanine nucleotide triphosphatase; GEF, guanine nucleotide exchange factor; EMT, epithelial-mesenchymal transition

Key words: receptor for advanced glycation end products, dedicator of cytokinesis protein $7, \mathrm{Cdc} 42$, filopodia, migration of RAGE-mediated cellular migration through the formation of dendritic pseudopodia.

\section{Introduction}

The receptor for advanced glycation end products (RAGE) is a type I transmembrane receptor belonging to the immunoglobulin superfamily. The receptor is involved in the pathogenesis of a broad range of inflammatory, degenerative and hyperproliferative diseases $(1,2)$. It binds to diverse ligands, including advanced glycation end products (AGEs) (3), high-mobility group box 1 (HMGB1) (4,5) and S100 family proteins (6). Binding of ligands to RAGE results in the activation of multiple intracellular signaling pathways, eventually leading to apoptosis, hyperproliferation, production of inflammatory cytokines and increased motility $(2,5,7)$.

Our previous study demonstrated that the cytoplasmic domain of RAGE is phosphorylated at Ser391 by PKC $\zeta$ upon the binding of ligands (12). TIRAP and MyD88 are recruited to the phosphorylated RAGE and transduce a signal to downstream effector molecules, such as NF- $\mathrm{B}$, Akt, p38 and JNK1. This finding may assist in elucidating the mechanisms of the various RAGE-mediated cellular processes.

Hudson et al (8) reported that diaphanous-1 (Dia-1) also functions as an adaptor for the RAGE cytoplasmic domain. This protein plays a major role in the control of the RAGE-mediated activation of the small guanine nucleotide triphosphatases (GTPases), Rac1 and Cdc42. In this pathway, the effector small GTPases are responsible for the formation of lamellipodia and filopodia, in turn facilitating cellular migration $(9,10)$. However, it remains unclear how the ligandbound RAGE leads to the activation of Rac-1 and Cdc42. For the activation of the small GTPases, involvement of a guanine nucleotide exchange factor (GEF) is essential, since GEFs catalyze the replacement of GDP with free cytoplasmic GTP to generate active GTPases. There have been no reports demonstrating that Dia-1 has GEF activity and a GEF-like domain is not present in Dia-1. 
In the present study, we aimed to identify a possible GEF(s) that is involved in the RAGE-Rac1/Cdc42 signaling axis. Our efforts resulted in the identification of an atypical DOCK180related GEF, dedicator of cytokinesis protein 7 (DOCK7). DOCK7 bound to the cytoplasmic domain of RAGE and the downregulation of DOCK7 resulted in marked interference with signal transduction from RAGE to Cdc42, indicating the involvement of DOCK 7 in the RAGE-Cdc42 signaling axis in human glioblastoma cells.

\section{Materials and methods}

Cell lines. The following human cancer cell lines were used: HEK293T (embryonic kidney cell line stably expressing the SV40 large T antigen; Riken BioResource Center, Tsukuba, Japan), SH-SY5Y (neuroblastoma; ATCC, Manassas, VA), U-87MG (glioblastoma; ATCC), PK-8 (pancreatic carcinoma; Cell Resource Center for Biomedical Research, Tohoku University, Sendai, Japan), HepG2 (hepatocellular carcinoma; ATCC), Hep3B (hepatocellular carcinoma; ATCC), HeLa (cervix adenocarcinoma; ATCC), PC-3 (prostate adenocarcinoma; ATCC), LNCaP (prostate carcinoma; ATCC), DU145 (prostate carcinoma; ATCC), A431 (skin epidermoid carcinoma; ATCC), HCT116 (colorectal carcinoma; ATCC), DLD-1 (colorectal adenocarcinoma; ATCC), KPK1 (renal clear cell carcinoma; Clonetics, San Diego, CA), Caki-1 (renal clear cell carcinoma; ATCC), Caki-2 (renal clear cell carcinoma; ATCC) and MCF7 (mammary gland adenocarcinoma; ATCC). These cell lines were cultivated in $\mathrm{D} / \mathrm{F}$ medium (Invitrogen, Carlsbad, CA) supplemented with 10\% FBS.

Liquid chromatography coupled with electrospray tandem mass spectrometry ( $L C$-MS/MS). Immunoprecipitated proteins were identified using a shotgun-type protein identification approach as previously described (11). Briefly, we composed a nano-flow liquid chromatography-mass spectrometry platform consisting of a Nanospace SI-2 high-performance liquid chromatography modified to adjust flow rate (Shiseido Co., Tokyo, Japan), a nano-electrospray ionization source modified according to Washburn et al (12) and an LTQ-Orbitrap hybrid mass spectrometer (Thermo Fisher Scientific, San Jose, CA) to obtain tandem mass spectra of tryptic peptides. The resulting tandem mass spectrometry spectra were analyzed using the Sequest algorithm against a non-redundant human protein database (NCBI, Feb 2007) for putative protein identification.

Plasmids. We prepared two mammalian expression vectors. The CMV promoter-intron (CMVi) from the phCMV-FSR ${ }^{\mathrm{TM}}$ vector (Genlantis, San Diego, CA) was inserted into the Promoterless pDNR-1r vector (Clontech-Takara, Mountain View, CA) and this was named pDNR-CMVi. The CMVi with a part of the HTLV type 1 LTR (RU5') was integrated into the pIDTSmart vector (Integrated Device Technology, San Jose, CA) and this was named pIDT-CMViR. Both vectors efficiently express short cargo cDNAs.

Human cDNAs encoding constitutively active types of HRAS (G12V), RHOA (G14V), RAC1 (G12V) and CDC42 (G12V) were conjugated with green fluorescent protein (GFP) at the N-terminal side and inserted into the pDNR-CMVi vector.
Human cDNAs encoding wild-type (WT) cytoplasmic domain (364-404 aa) lacking a dominant-negative (DN) form and a cytoplasmic domain (Cyt: 364-404 aa) of RAGE were inserted into the pIDT-CMViR vector (13). WT, DN and Cyt of RAGE were designed to be expressed as C-terminal Myc-HAFlag-6His-tagged forms. Human cDNA encoding S100B was also tagged with C-terminal Myc-6His and inserted into the pIDT-CMViR vector.

Transient transfection of the plasmids into cultured cells was performed using FuGENE HD (Promega Biosciences, San Luis Obispo, CA) for HEK293T, U-87MG and PC3 cells and TransIT-keratinocyte (Mirus Bio LLC, Madison, WI) for MCF7 cells.

Western blot analysis and co-immunoprecipitation. Western blot analysis was performed under conventional conditions. Antibodies used were as follows: mouse anti-HA tag (clone 6E2), rabbit anti-human RAGE (Santa Cruz Biotechnology, Santa Cruz, CA), rabbit anti-human DOCK7 (Sigma-Aldrich, St. Louis, MO) and mouse anti-human tubulin antibodies (Sigma-Aldrich). The secondary antibodies were horseradish peroxidase-conjugated anti-mouse or anti-rabbit IgG antibody (Cell Signaling Technology, Beverly, MA). Positive signals were detected by a chemiluminescence system (ECL Plus; GE Healthcare Bio-Sciences, Piscataway, NJ).

Rabbit anti-human RAGE antibody was biotinylated using a Biotin Labeling kit-SH (Dojindo Molecular Technologies, Rockville, MD) to recover antibody-free RAGE after immunoprecipitation using streptavidin-agarose (13). Monoclonal anti-HA (clone HA-7)-tagged agarose (Sigma-Aldrich) and streptavidin agarose (Invitrogen) were used for the co-immunoprecipitation experiments. Monoclonal anti-His tag (clone 2D8) agarose (MBL, Nagoya, Japan) was used to purify recombinant human S100B protein expressed in the HEK293T cells.

The GTP-bound form of Cdc42 was determined using a Rac/Cdc42 Activation Assay kit (Millipore, Billerica, MA).

SiRNA. Human RAGE (siRAGE: siGENOME SMARTpool M-003625-02-0005), human DOCK7 (siDOCK7: siGENOME SMART pool M-031725-01-0005) and the negative control (siCont: siGENOME non-targeting siRNA pool \#1, D-001210-01) siRNAs were purchased from Thermo Scientific Dharmacon (Lafayette, CO). The siRNAs (100 nM) were transfected using Lipofectamine RNAiMax reagent (Invitrogen).

Migration assay. Migration of human glioblastoma U-87MG cells was assayed using a 24-well disposable chemotaxis system (cell culture inserts, $8-\mu \mathrm{m}$ pore size; BD Falcon, Franklin Lakes, NJ). The lower wells of the chamber were loaded with DMEM supplemented with $10 \%$ FBS. Cells $(5,000)$ were placed in the upper wells under a serum-free condition. After incubation for $8 \mathrm{~h}$, cells on the lower surface of the filter were counted after staining with hematoxylin and eosin $(H \& E)(n=4)$.

Immunocytochemistry. To visualize overexpressed RAGE, fixed cells were treated with rabbit anti-HA antibody (MBL) at RT for $1 \mathrm{~h}$ and were further treated with Alexa 594-conjugated 
A

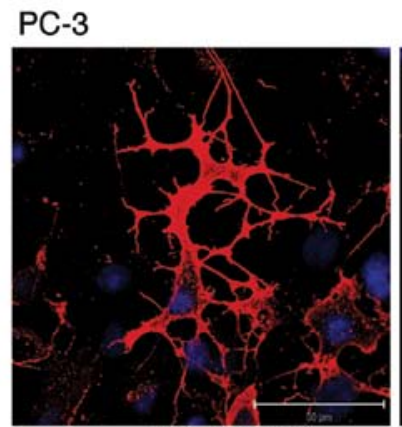

RAGE (WT)

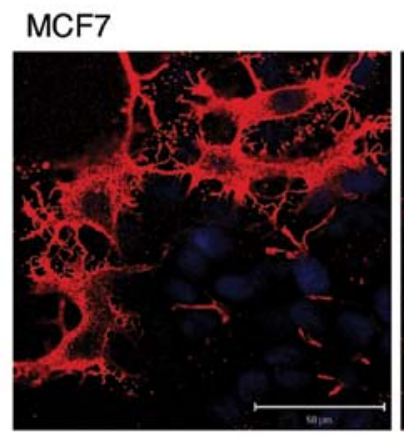

RAGE (WT)

U-87MG

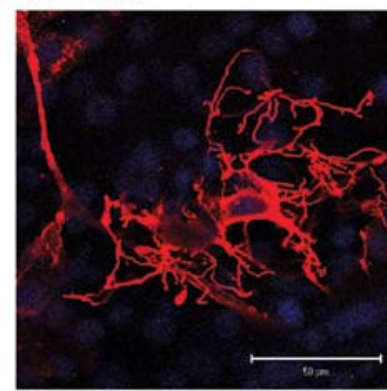

RAGE (WT)

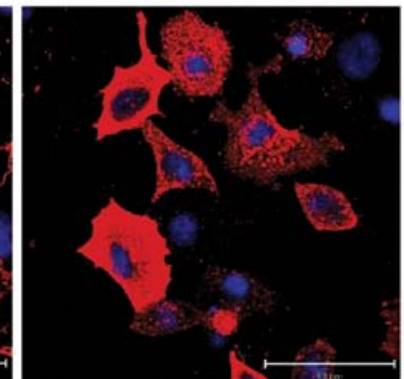

RAGE (DN)

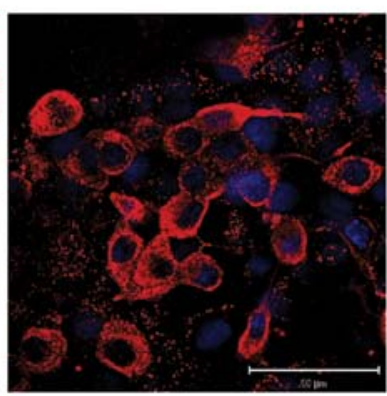

RAGE (DN)

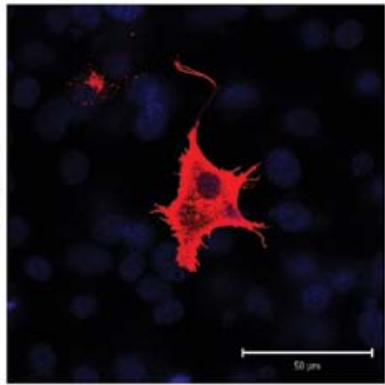

RAGE (DN)
B
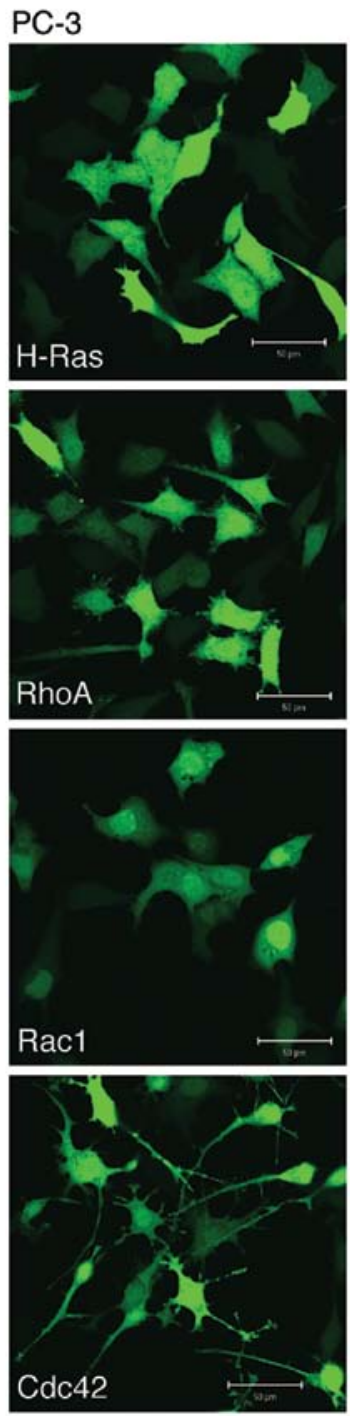

Figure 1. Forced expression of RAGE induces a typical morphological change in human cancer cells. (A) PC-3, MCF7 and U-87MG cells were transiently transfected with $5 \mu \mathrm{g}$ of a plasmid expressing wild-type (WT) or dominant-negative (DN) RAGE. After a 48-h incubation, cells expressing exogenous RAGE were identified by indirect immunostaining with anti-HA antibody (in red). Nuclei were stained with DAPI (in blue). (B) PC-3 cells were transfected with a plasmid expressing a green fluorescent protein (GFP)-fused constitutively active small GTPase, H-Ras, RhoA, Rac1 or Cdc42, under conditions similar to those in A. Successfully transfected cells were detected by GFP (in green). Scale bar, $50 \mu \mathrm{m}$.

goat anti-rabbit IgG antibody (Molecular Probes/Invitrogen, Eugene, OR) under the same conditions as those previously reported (14).

Statistical analysis. Data are expressed as the means \pm SD. We employed simple pair-wise comparison with the Student's t-test (two-tailed distribution with two-sample equal variance). $\mathrm{P}<0.05$ was considered to indicate a statistically significant difference.

\section{Results}

Overexpression of RAGE induces the formation of dendritic pseudopodia in cancer cells. It has been shown that upon ligand binding RAGE activates Rac1 and Cdc42 (8), eventually leading to the induction of lamellipodial and filopodial formation, respectively $(15,16)$. We, therefore, examined which morphological changes were mainly induced by RAGE. Overexpression of full-length WT RAGE, but not that of cytoplasmic domain-deleted DN type of RAGE, induced dramatic morphological changes in all human cancer cell lines examined (Fig. 1A). The most typically observed morphological change was the presence of highly branched filopodia-like protrusions (dendritic pseudopodia). When various small GTPases were overexpressed in PC-3 cells, Cdc42 induced a morphological change most similar to that induced by WT RAGE when compared to Ras, Rho and Racl (Fig. 1B), suggesting that the formation of dendritic pseudopodia is due to the activation of the RAGE-Cdc42 signaling axis.

DOCK7 binds to the RAGE cytoplasmic domain. We aimed to identify a candidate protein(s) involved in mediating a signal from RAGE to Cdc42. We screened proteins that bound to the overexpressed RAGE cytoplasmic domain in HEK293T 


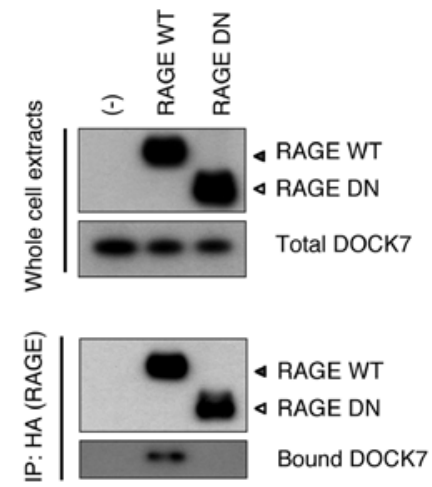

Figure 2. DOCK7 binds to the RAGE cytoplasmic domain. HEK293T cells were transfected with wild-type (WT) and dominant-negative (DN) RAGE tagged with Myc-HA-Flag-6His $(5 \mu \mathrm{g})$. Forty-eight hours after transfection, cell extracts were analyzed by western blot analysis with or without immunoprecipitation (IP) using the anti-HA antibody.

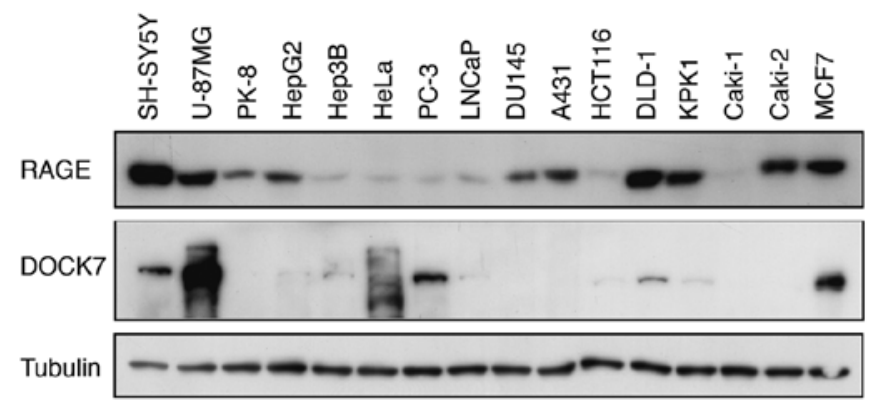

Figure 3. Expression profiles of RAGE and DOCK7 in human cancer cell lines. Extracts of various cell lines were analyzed for the expression of RAGE and DOCK7 by western blot analysis. Tubulin was used as a control for amounts of loaded protein.
A

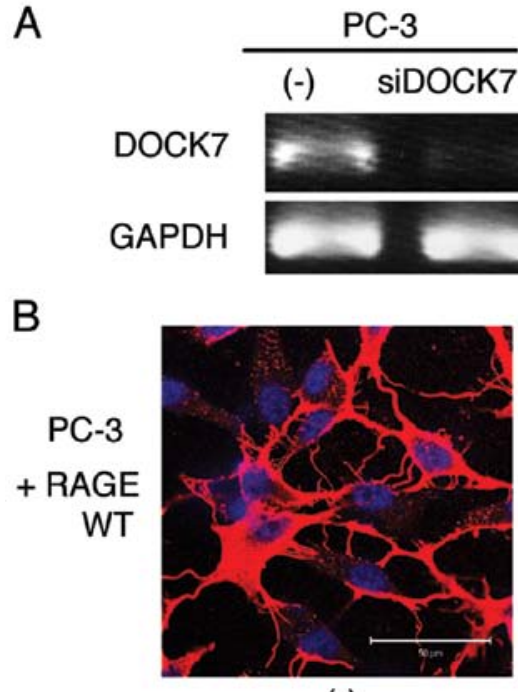

$(-)$

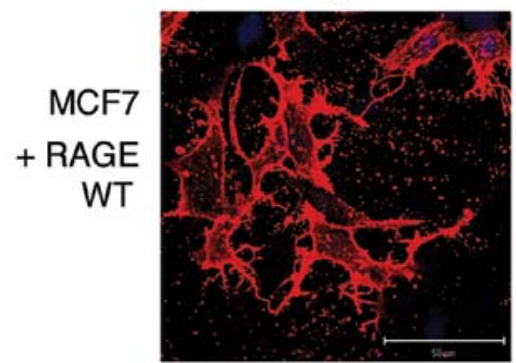

$(-)$

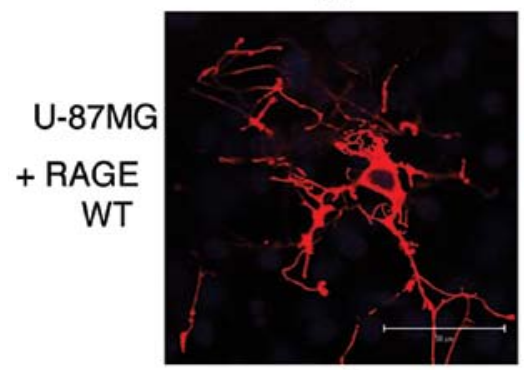

$(-)$
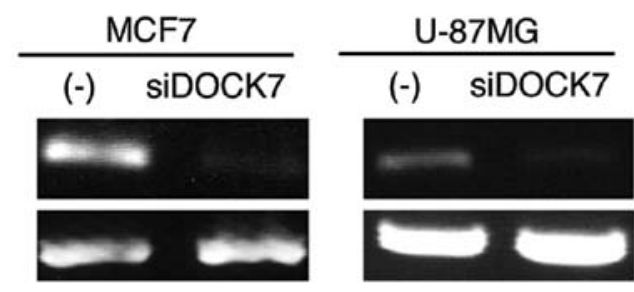

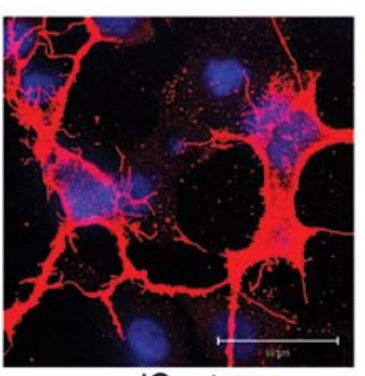

+ siCont.

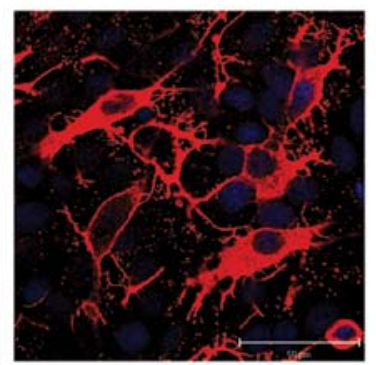

+ siCont.

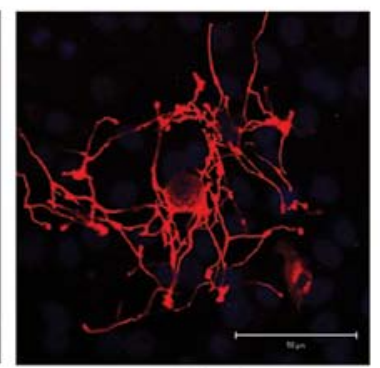

+ siCont.

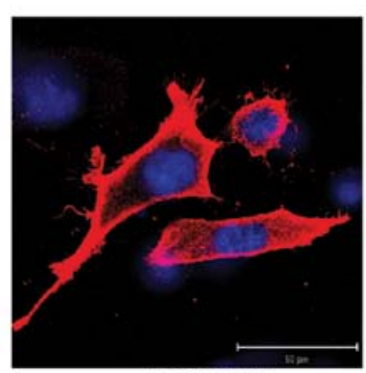

+ siDOCK7

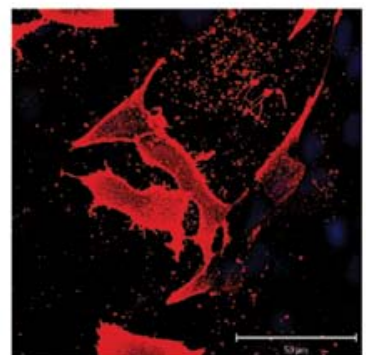

+ siDOCK7

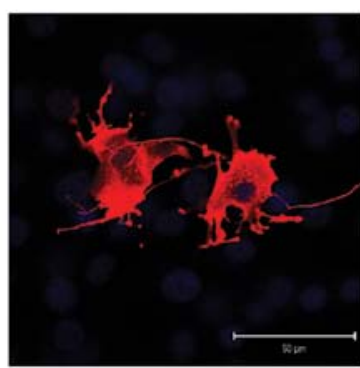

+ siDOCK7

Figure 4. DOCK7 plays a critical role in RAGE-mediated formation of dendritic pseudopodia in human cancer cells. (A) The inhibitory effect of siRNA $(100 \mathrm{nM})$ on DOCK7 expression was assessed by RT-PCR. Twenty-four hours after transfection with DOCK7 siRNA (100 nM), cells were transfected with a WT RAGE-expressing plasmid $(5 \mu \mathrm{g})$ and harvested $48 \mathrm{~h}$ later. (B) Cells were treated as described in A and examined under conditions similar to those described in the legend of Fig. 1A except for the downregulation of DOCK7 with siRNA. 


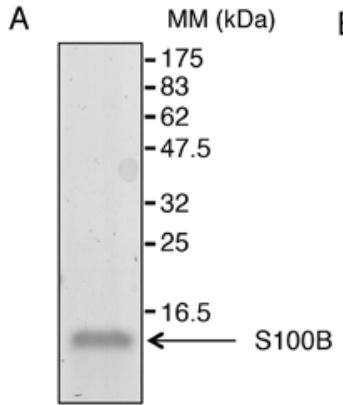

C

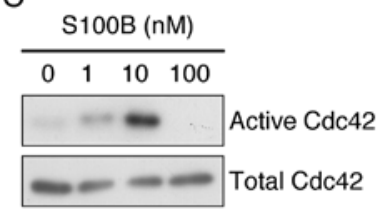

B $\quad \mathrm{S} 100 \mathrm{~B}(\mathrm{nM})$

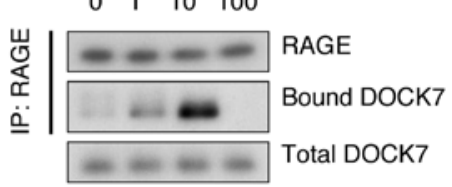

D
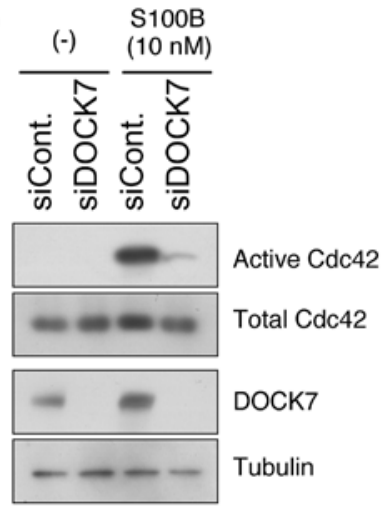

Figure 5. DOCK7 mediates a signal from RAGE to Cdc42. (A) Recombinant human S100B was purified from the serum-free conditioned medium of HEK293 cells that were transfected with a plasmid expressing S100B. The purified protein was subjected to SDS-PAGE and detected by Coomassie Brilliant Blue staining. (B) U-87MG cells were treated with the recombinant S100B for $10 \mathrm{~min}$. Binding of DOCK7 to RAGE was demonstrated by immunoprecipitation using biotinylated-RAGE antibody with streptavidin-agarose followed by western blot analysis using anti-RAGE and anti-DOCK7 antibodies. (C) Activation of Cdc42 (GTP-bound form) by S100B was assessed by an active $\mathrm{Cdc} 42$ detection system (Materials and methods). Cells were treated under conditions similar to those described in B. (D) Abrogation of S100B-induced activation of Cdc42 by the downregulation of DOCK7 with siRNA. U-87MG cells were treated with DOCK7 siRNA (100 nM) for $72 \mathrm{~h}$ prior to a 10-min stimulation with $\mathrm{S} 100 \mathrm{~B}(10 \mathrm{nM})$ and the activation of $\mathrm{Cdc} 42$ was assessed.

cells, employing immunoprecipitation and affinity purification followed by mass spectrometry. Among the proteins identified by mass spectrometry, Ras-GTPase-activating protein $\mathrm{SH} 3$ domain-binding proteins (G3BP) and DOCK7 were noted as promising. However, neither cloned and overexpressed G3BP nor endogenous G3BP were noted to bind to Cyt RAGE in transfected cells (data not shown). Endogenous DOCK7, the only GEF discovered in the analysis, was confirmed to bind to WT RAGE but not to DN RAGE in HEK293T cells (Fig. 2). This indicated that RAGE interacts with DOCK7 via its cytoplasmic domain.

Expression of RAGE and DOCK7 in human cancer cell lines. We next examined the expression of RAGE and DOCK7 in various human cancer cell lines by western blot analysis. RAGE protein was detected in all cancer cell lines examined and higher expression levels were noted in SH-SY5Y, U-87MG, PK-8, HepG2, DU145, A431, DLD-1, KPK1, Caki-2 and MCF7 cell lines (Fig. 3). DOCK7 protein was clearly detected in SH-SY5Y, U-87MG, HeLa, PC-3, DLD-1 and MCF7 cell lines with the highest expression level in U-87MG. Thus, the notion that the induction of dendritic pseudopodia
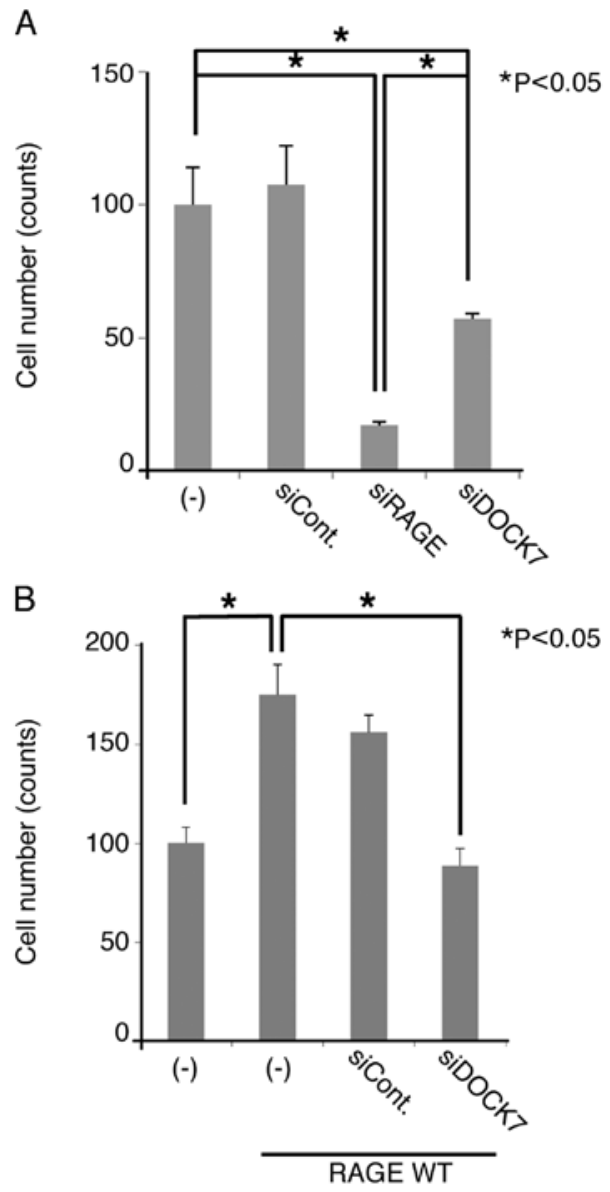

Figure 6. Migration of U-87MG cells in vitro depends on RAGE and its downstream effector DOCK7. (A) Migration of U-87MG cells was inhibited by the downregulation of RAGE or DOCK7 with siRNA (100 nM). Cells were transfected with siRNAs $(100 \mathrm{nM}) 72 \mathrm{~h}$ prior to re-inoculation into culture inserts (upper chamber, serum-free; lower chamber, 10\% FBS) and cells having migrated to the lower surface were determined $8 \mathrm{~h}$ later. (B) Abrogation of RAGE-induced migration of U-87MG cells by DOCK7 siRNA (100 nM). WT RAGE was transfected $24 \mathrm{~h}$ prior to application with siRNA to U-87MG cells, which were analyzed under conditions similar to those in A.

formation by overexpressed WT RAGE (Fig. 1) is mediated by DOCK7 appeared plausible.

Knockdown of DOCK7 suppresses formation of dendritic pseudopodia in RAGE-overexpressed cells. Using the DOCK7-positive cell lines, U-87MG, MCF7 and PC3, we examined the possible role of DOCK7 in the formation of dendritic pseudopodia induced by RAGE overexpression. Application of DOCK7 siRNA efficiently suppressed the expression of endogenous DOCK7 (Fig. 4A). The validated siRNA markedly inhibited the RAGE-induced formation of dendritic pseudopodia, while the control siRNA demonstrated no effect (Fig. 4B).

A RAGE ligand, $S 100 B$, induces the recruitment of DOCK7 to the RAGE cytoplasmic domain and activates Cdc42. S100B is a physiological ligand of RAGE that is abundantly produced and secreted by glioblastoma cells (17). We prepared highly purified recombinant human $\mathrm{S} 100 \mathrm{~B}$ using a mammalian expression system (Fig. 5A, Materials and methods). When 
U-87MG cells were treated with different concentrations of S100B, DOCK7 was recruited to endogenous RAGE in a dose-dependent manner up to $10 \mathrm{nM}$, while $100 \mathrm{nM}$ of S100B abolished the binding possibly due to a cytotoxic effect (Fig. 5B). In accordance with this, Cdc42 was dosedependently activated up to $10 \mathrm{nM}$ and inactivated at $100 \mathrm{nM}$ of S100B (Fig. 5C). In addition, siRNA-mediated downregulation of DOCK7 effectively abrogated the activation of Cdc42 caused by S100B in U-87MG cells (Fig. 5D). These results indicate that DOCK7 is an essential mediator of the RAGE-Cde42 signaling axis.

Knockdown of DOCK7 suppresses the migration of $U-87 M G$ cells. Since the RAGE-Cdc42 signaling axis is considered to play a critical role in cellular migration (8), we examined the effect of DOCK7 siRNA on the migration of U-87MG cells. When we treated the cells with RAGE and DOCK7 siRNAs, cellular migration was significantly suppressed (Fig. 6A), indicating that endogenous RAGE and DOCK7 are involved in the migration. The extent of suppression by the downregulation of RAGE was greater compared to that of DOCK7 siRNA (Fig. 6A). Overexpression of RAGE enhanced the migration of U87MG cells, this being abrogated by DOCK7 siRNA (Fig. 6B). These results indicate that DOCK7 functions as an essential and downstream regulator of RAGE-mediated cellular migration in $\mathrm{U}-87 \mathrm{MG}$ cells

\section{Discussion}

Several lines of evidence indicate that RAGE activation upon ligand binding promotes cancer progression by enhancing cell proliferation and migration (4). In this study, we focused on migration enhanced by the RAGE signaling pathway. Rho family small GTPases are widely accepted as key regulators of cellular migration via modulation of the structural network of actin cytoskeleton and the eventual formation of various pseudopodia. The resemblance of the morphological change of RAGE-transfected cells with Cdc42-induced morphology (Fig. 1) indicated that $\mathrm{Cdc} 42$ rather than Rac1 may be involved in the RAGE signaling in cancer cells.

Hudson et al (8) demonstrated that Dia-1 functions as an adaptor protein for RAGE and transfers a signal from RAGE to Rac1 and Cdc42, eventually enhancing cellular migration. Despite the essential role of Dia-1 in this process, it remains unclear how Dia-1 activates Rac1 and Cdc42. It is possible that an adaptor GEF(s) plays a role in the activation of Rac1 and Cdc42. No obvious domains or motifs associated with guanyl-nucleotide exchange function were observed in Dia-1. We successfully identified DOCK7 using Cyt RAGE as a bait. Since it is difficult to efficiently express a short cDNA such as Cyt RAGE (41 aa), we modified the mammalian expression vectors as described in Materials and methods. It is difficult to purify full-length recombinant DOCK7 protein due to its high molecular weight $(2,140 \mathrm{aa})$. Hence, we confirmed the binding of DOCK7 to Cyt RAGE by immunoprecipitation using the cell extracts (Figs. 2 and 5B and D). This did not provide an answer as to whether the binding is direct or indirect. It is possible, therefore, that Dia-1 functions as a mediator between RAGE and DOCK7. We observed that the downregulation of Dia-1 by siRNA abrogated the formation of dendritic pseudopodia in RAGE-overexpressed cancer cells (data not shown).

DOCK7 belongs to the DOCK family, which consists of eleven GEFs (18). DOCK proteins contain a catalytic domain termed the DOCK homology region (DHR)-2 (19). Although the molecular structures of DOCK proteins are similar, the small GTPases, including Rac and $\mathrm{Cdc} 42$, are regulated by specific DOCK proteins (18). DOCK180, DOCK2 and DOCK3 are Rac-specific GEFs. DOCK4 and DOCK5 are structurally deduced GEFs for Rac. DOCK6, DOCK7 and DOCK8 are GEFs for Rac and Cdc42. DOCK9, DOCK10 and DOCK11 are Cdc42-specific GEFs. It is also known that each DOCK protein is differentially expressed in different cell types (18). We, therefore, examined DOCK7 expression in various cancer cell lines. DOCK7 was detected in a number of cancer cell lines with varying expression levels. Among those, U-87MG cells demonstrated the highest level of DOCK7 expression (Fig. 3). This may influence the RAGE-Cdc42 signaling axis in different cancer types. In fact, the overexpression of RAGE induced no marked morphological change in DOCK7-deficient PK-8 cells (data not shown). Thus, we reasonably conclude that DOCK7 binding to RAGE leads to Cdc42 activation at least in several types of cancer cells.

It should be noted that siRNA-mediated downregulation of RAGE suppressed migration of U-87MG cells more effectively when compared to that of DOCK7 (Fig. 6A). This may imply the involvement of factors other than DOCK7 in the RAGE-mediated migration. We recently demonstrated that signal transduction triggered by RAGE is partially mediated by the adaptor proteins TIRAP and MyD88 (13). The RAGETIRAP/MyD88 signaling axis leads to the activation of NF- $\mathrm{KB}$, resulting in the expression of genes related to the inflammatory response, including TNF- $\alpha$ and IL-6. Several studies have shown that the MyD88 signaling pathway plays a crucial role in neutrophil migration $(20,21)$. Dia-1 may mediate a signal from RAGE to downstream mediators other than DOCK7. Thus, the RAGE-induced cellular migration may be controlled by more complicated mechanisms involving not only DOCK7 but also MyD88, Dia-1 and yet unidentified molecules.

NF- $\mathrm{kB}$ has been shown to be a principal mediator for RAGE signaling in various biological contexts such as inflammation and stress responses (22). Recently, accumulating evidence indicates that NF- $\kappa B$ is critically involved in epithelial-mesenchymal transition (EMT), a complex reprogramming process of epithelial cells that plays an indispensable role in cancer invasion and metastasis. Therefore, it is possible that NF- $\mathrm{kB}-$ mediated EMT and DOCK7-mediated migration coordinately regulate cancer invasion and metastasis in response to RAGE activation in inflammatory microenvironments.

\section{Acknowledgements}

The present study was supported in part by grants from the Ministry of Health, Labor and Welfare (Research for Intractable Diseases) (to N.H.), the Ministry of Education, Culture, Sports, Science and Technology of Japan (Grantin-Aid for Scientific Research on Innovation Areas) (to M.S.), The Naito Foundation (to M.S.), the Senshin Medical Research Foundation (to M.S.) and the Research Foundation for Pharmaceutical Sciences (to M.S.). 


\section{References}

1. Yan SF, Ramasamy R and Schmidt AM: Mechanisms of disease: advanced glycation end-products and their receptor in inflammation and diabetes complications. Nat Clin Pract Endocrinol Metab 4: 285-293, 2008.

2. Lin L, Park S and Lakatta EG: RAGE signaling in inflammation and arterial aging. Front Biosci 14: 1403-1413, 2009.

3. Xue J, Rai V, Singer D, Chabierski S, Xie J, Reverdatto S, Burz DS, Schmidt AM, Hoffmann R and Shekhtman A: Advanced glycation end product recognition by the receptor for AGEs. Structure 19: 722-732, 2011

4. Taguchi A, Blood DC, del Toro G, Canet A, Lee DC, Qu W, Tanji N, Lu Y, Lalla E, Fu C, Hofmann MA, Kislinger T, Ingram M, Lu A, Tanaka H, Hori O, Ogawa S, Stern DM and Schmidt AM: Blockade of RAGE-amphoterin signalling suppresses tumour growth and metastases. Nature 405: 354-360, 2000.

5. Sims GP, Rowe DC, Rietdijk ST, Herbst R and Coyle AJ: HMGB1 and RAGE in inflammation and cancer. Annu Rev Immunol 28: 367-388, 2010.

6. Leclerc E, Fritz G, Vetter SW and Heizmann CW: Binding of S100 proteins to RAGE: an update. Biochim Biophys Acta 1793: 993-1007, 2009.

7. Gebhardt C, Riehl A, Durchdewald M, Németh J,Fürstenberger G, Müller-Decker K, Enk A, Arnold B, Bierhaus A, Nawroth PP, Hess J and Angel P: RAGE signaling sustains inflammation and promotes tumor development. J Exp Med 205: 275-285, 2008.

8. Hudson BI, Kalea AZ, Del Mar Arriero M, Harja E, Boulanger E, D'Agati V and Schmidt AM: Interaction of the RAGE cytoplasmic domain with diaphanous-1 is required for ligand-stimulated cellular migration through activation of Rac1 and Cdc42. J Biol Chem 283: 34457-34468, 2008.

9. Fukata M, Nakagawa M and Kaibuchi K: Roles of Rho-family GTPases in cell polarisation and directional migration. Curr Opin Cell Biol 15: 590-597, 2003.

10. Leve F and Morgado-Díaz JA: Rho GTPase signaling in the development of colorectal cancer. J Cell Biochem 113: 2549-2559, 2012.

11. Motoyama A and Yates JR III: Multidimensional LC separations in shotgun proteomics. Anal Chem 80: 7187-7193, 2008.

12. Washburn MP, Wolters D and Yates JR III: Large-scale analysis of the yeast proteome by multidimensional protein identification technology. Nat Biotechnol 19: 242-247, 2001.
13. Sakaguchi M, Murata H, Yamamoto K, Ono T, Sakaguchi Y, Motoyama A, Hibino T, Kataoka K and Huh NH: TIRAP, an adaptor protein for TLR2/4, transduces a signal from RAGE phosphorylated upon ligand binding. PLoS One 6: e23132, 2011.

14. Sakaguchi M, Miyazaki M, Takaishi M, Sakaguchi Y, Makino E, Kataoka N, Yamada H, Namba M and Huh NH: S100C/A11 is a key mediator of $\mathrm{Ca}\left(2^{+}\right)$-induced growth inhibition of human epidermal keratinocytes. J Cell Biol 163: 825-835, 2003.

15. Huttunen HJ, Fages $\mathrm{C}$ and Rauvala $\mathrm{H}$ : Receptor for advanced glycation end products (RAGE)-mediated neurite outgrowth and activation of NF-kappaB require the cytoplasmic domain of the receptor but different downstream signaling pathways. J Biol Chem 274: 19919-19924, 1999.

16. Wang L, Li S and Jungalwala FB: Receptor for advanced glycation end products (RAGE) mediates neuronal differentiation and neurite outgrowth. J Neurosci Res 86: 1254-1266, 2008.

17. Davey GE, Murmann P and Heizmann CW: Intracellular $\mathrm{Ca}^{2+}$ and $\mathrm{Zn}^{2+}$ levels regulate the alternative cell density-dependent secretion of S100B in human glioblastoma cells. J Biol Chem 276: 30819-30826, 2011.

18. Miyamoto Y and Yamauchi J: Cellular signaling of Dock family proteins in neural function. Cell Signal 22: 175-182, 2010.

19. Premkumar L, Bobkov AA, Patel M, Jaroszewski L, Bankston LA, Stec B, Vuori K, Côté JF and Liddington RC: Structural basis of membrane targeting by the Dock 180 family of Rho family guanine exchange factors (Rho-GEFs). J Biol Chem 285: 13211-13222, 2010

20. Zhelev DV and Alteraifi A: Signaling in the motility responses of the human neutrophil. Ann Biomed Eng 30: 356-370, 2002.

21. Castoldi A, Braga TT, Correa-Costa M, Aguiar CF, Bassi Ê J, Correa-Silva R, Elias RM, Salvador F, Moraes-Vieira PM, Cenedeze MA, Reis MA, Hiyane MI, Pacheco-Silva Á, Gonçalves GM and Câmara NO: TLR2, TLR4 and the MYD88 signaling pathway are crucial for neutrophil migration in acute kidney injury induced by sepsis. PLoS One 7: e37584, 2012.

22. Li CW, Xia W, Huo L, Lim SO, Wu Y, Hsu JL, Chao CH, Yamaguchi H, Yang NK, Ding Q, Wang Y, Lai YJ, LaBaff AM, Wu TJ, Lin BR, Yang MH, Hortobagyi GN and Hung MC: Epithelial-mesenchymal transition induced by TNF- $\alpha$ requires NF- $\kappa B$-mediated transcriptional upregulation of Twist1. Cancer Res 72: 1290-1300, 2012. 